\title{
Relativistic frozen core potential scheme with relaxation of core electrons
}

\author{
Yuya Nakajima ${ }^{\mathrm{a}}$, Junji Seino ${ }^{\mathrm{b}}$, Masao Hayami ${ }^{\mathrm{a}}$, Hiromi Nakai ${ }^{\mathrm{a}, \mathrm{b}, \mathrm{c}, \mathrm{d}, *}$ \\ ${ }^{a}$ Department of Chemistry and Biochemistry, School of Advanced Science and Engineering, Waseda University, \\ Tokyo 169-8555, Japan \\ ${ }^{b}$ Research Institute for Science and Engineering, Waseda University, Tokyo 169-8555, Japan \\ ${ }^{c}$ CREST, Japan Science and Technology Agency, 4-1-8 Honcho, Kawaguchi, Saitama 332-0012, Japan \\ ${ }^{d}$ Elements Strategy Initiative for Catalysts and Batteries (ESICB), Kyoto University, Katsura, Kyoto 615-8520, Japan
}

\begin{abstract}
This letter proposes a relaxation scheme for core electrons based on the frozen core potential method at the infinite-order Douglas-Kroll-Hess level, called FCP-CR. The core electrons are self-consistently relaxed using frozen molecular valence potentials after the valence SCF calculation is performed. The efficiency of FCP-CR is confirmed by calculations of gold clusters. Furthermore, FCP-CR reproduces the results of the all-electron method for the energies of coinage metal dimers and the core ionization energies and core level shifts of vinyl acetate and three tungsten complexes at the Hartree-Fock and/or symmetry-adapted cluster configuration interaction levels.
\end{abstract}

*Corresponding author at: Department of Chemistry and Biochemistry, School of Advanced Science and Engineering, Waseda University, Tokyo 169-8555, Japan. Fax: +8133205 2504.

Email address: nakai@waseda.jp (Hiromi Nakai)

Preprint submitted to Chemical Physics Letter

September 23, 2016

(C) 2016. This manuscript version is made available under the Elsevier user license 


\section{Introduction}

2

3 odic table is one of the most significant problems in quantum chemistry. One rigorous scheme

4 is the four-component formalism based on the Dirac equation, which satisfies the Lorentz invari-

5 ance for the motion of electrons [1]. This scheme automatically includes both the spin-free and

6 spin-dependent relativistic effects. Recent theoretical and computational developments in the four-

7 component method have enlarged its applicability for treating large molecules [2-4]. An alterna-

The development of a highly accurate and efficient scheme to treat any elements in the peritive decade, several schemes have been proposed, such as the normalized elimination of small component [5], exact two-component method [6], and infinite-order Douglas-Kroll-Hess (IODKH) [7] methods, which provide the same energies as the four-component method. From the viewpoint of efficiency in two-component formalisms, the locality of the relativistic effect has been considered [8-16]. Our group has also proposed a scheme, termed local unitary transformation (LUT), for both one- and two-electron relativistic transformations $[10,11]$. Furthermore, the LUT scheme was combined with the divide-and-conquer method to achieve an overall linear-scaling technique from integral calculations to post-Hartree-Fock (HF) calculations [12-14]. These improvements can reduce the gap between non-relativistic and highly accurate relativistic methods in all-electron (AE) calculations.

A widely used method to reduce the number of electrons treated explicitly is the effective core 
potential (ECP) method. The ECP method, which includes the pseudo-potential (PP) [17-23] and model potential (MP) [24-27] methods, replaces the effect of the core electrons with a core potential. The ECP method includes part of the relativistic effects in the core potential. The PP method introduces a smooth and nodeless potential function in the core region. On the other hand, the MP method uses a nodal potential function to describe the valence orbital in the AE treatment. Our group has also proposed another scheme for reducing the number of electrons treated explicitly, which is termed the frozen core potential (FCP) method [28]. The FCP method is based on the same equation as the MP method but the core electrons obtained from atomic calculations are treated explicitly. Another advantage of the FCP method is a seamless connection to the AE method with an accurate two-component relativistic Hamiltonian. The ECP and FCP methods are quite effective for determining valence electronic states and valence properties. However, the ECP method has difficulties in describing core electron states and their properties because the core potential is obtained by atomic calculations without the consideration of any chemical environment effect.

In the present study, we propose a novel scheme based on the FCP method to describe core properties using the relaxations of core electrons. This letter is organized as follows; the theory and implementation are described in Sec. 2; numerical assessments are performed in Sec. 3; and concluding remarks are given in Sec. 4. 


\section{Theory and implementation}

\subsection{FCP with relaxation of core electrons}

This subsection provides the theory for core electron relaxations based on the FCP scheme.

The FCP method is based on the Huzinaga-Cantu equation [29], which separates electrons into core and valence regions. Here, we discuss a closed-shell system for simplicity, though extension to an open-shell system is straightforward. The AE HF-Roothaan (HFR) equation is given by

$$
\mathbf{F}(\mathbf{D}) \mathbf{C}=\mathbf{S C E}
$$

where $\mathbf{F}, \mathbf{D}, \mathbf{S}, \mathbf{C}$, and $\mathbf{E}$ are the Fock, density, overlap, orbital coefficient, and orbital energy matrices. The Fock matrix is written as

$$
F_{\mu \nu}(\mathbf{D})=H_{\mu \nu}+G_{\mu \nu}
$$

and

$$
D_{\mu \nu}=2 \sum_{i}^{\mathrm{occ}} C_{\mu i} C_{v i}^{*} .
$$

Here, $H_{\mu \nu}$ and $G_{\mu \nu}$ are non-relativistic (NR)/relativistic one- and two-electron integrals with respect to atomic orbitals $\{\mu, \nu\}$, respectively. The density matrix $\mathbf{D}$ is separated into core and valence contributions as

$$
\mathbf{D}=\mathbf{D}^{\mathrm{C}}+\mathbf{D}^{\mathrm{V}} .
$$

In the valence FCP method, two approximations are adopted. First, the total density matrix $\mathbf{D}$ 
is approximated in a block-diagonal form:

where $\mathbf{d}^{\mathrm{C}}\left(\mathbf{d}^{\mathrm{V}}\right)$ is a submatrix that includes only the core (valence) AO indices. This approximation is effective if a generally contracted basis set is adopted. Second, the molecular core density matrix $\mathbf{D}^{\mathrm{C}}$ is approximated as the direct sum of the atomic contributions as follows,

60

$$
\mathbf{d}^{\mathrm{C}} \approx \tilde{\mathbf{d}}^{\mathrm{C}}=\tilde{\mathbf{d}}_{A}^{\mathrm{C}} \oplus \tilde{\mathbf{d}}_{B}^{\mathrm{C}} \oplus \cdots
$$

$$
\begin{aligned}
f_{\mu \nu}^{\mathrm{V}}= & h_{\mu \nu}^{\mathrm{V}}+\frac{1}{2} \sum_{\lambda, \rho} d_{\lambda \rho}^{\mathrm{V}}\left(\left\langle\chi_{\mu}^{\mathrm{V}} \chi_{\lambda}^{\mathrm{V}} \mid \chi_{\nu}^{\mathrm{V}} \chi_{\rho}^{\mathrm{V}}\right\rangle-\frac{1}{2}\left\langle\chi_{\mu}^{\mathrm{V}} \chi_{\lambda}^{\mathrm{V}} \mid \chi_{\rho}^{\mathrm{V}} \chi_{\nu}^{\mathrm{V}}\right\rangle\right) \\
& +\frac{1}{2} \sum_{\lambda, \rho} \sum_{A}\left(\tilde{d}_{A}^{\mathrm{C}}\right)_{\lambda \rho}\left(\left\langle\chi_{\mu}^{\mathrm{V}} \chi_{\lambda}^{\mathrm{C}} \mid \chi_{\nu}^{\mathrm{V}} \chi_{\rho}^{\mathrm{C}}\right\rangle-\frac{1}{2}\left\langle\chi_{\mu}^{\mathrm{V}} \chi_{\lambda}^{\mathrm{C}} \mid \chi_{\rho}^{\mathrm{C}} \chi_{\nu}^{\mathrm{V}}\right\rangle\right) \\
& -2 \sum_{l, m} s_{\mu l} w_{l m}^{\mathrm{C}} s_{m v}
\end{aligned}
$$

and

$$
w_{\mu \nu}^{\mathrm{C}}=\sum_{c}^{\text {core }} \varepsilon_{c} c_{\mu c} c_{v c}^{*},
$$

where $\left\langle\chi_{\mu} \chi_{\lambda} \mid \chi_{\nu} \chi_{\rho}\right\rangle$ is the two-electron integral, $\mathbf{S}$ is the overlap matrix, $\varepsilon_{c}$ is the core orbital energy, and $\left\{c_{\mu c}\right\}$ is the core orbital coefficient. The last term is a projection operator that prevents electrons 


$$
\begin{aligned}
f_{\mu \nu}^{\mathrm{C}}= & h_{\mu \nu}^{\mathrm{C}}+\frac{1}{2} \sum_{\lambda, \rho} d_{\lambda \rho}^{\mathrm{C}}\left(\left\langle\chi_{\mu}^{\mathrm{C}} \chi_{\lambda}^{\mathrm{C}} \mid \chi_{\nu}^{\mathrm{C}} \chi_{\rho}^{\mathrm{C}}\right\rangle-\frac{1}{2}\left\langle\chi_{\mu}^{\mathrm{C}} \chi_{\lambda}^{\mathrm{C}} \mid \chi_{\rho}^{\mathrm{C}} \chi_{\nu}^{\mathrm{C}}\right\rangle\right) \\
& +\frac{1}{2} \sum_{\lambda, \rho} d_{\lambda \rho}^{\mathrm{V}}\left(\left\langle\chi_{\mu}^{\mathrm{C}} \chi_{\lambda}^{\mathrm{V}} \mid \chi_{\nu}^{\mathrm{C}} \chi_{\rho}^{\mathrm{V}}\right\rangle-\frac{1}{2}\left\langle\chi_{\mu}^{\mathrm{C}} \chi_{\lambda}^{\mathrm{V}} \mid \chi_{\rho}^{\mathrm{V}} \chi_{\nu}^{\mathrm{C}}\right\rangle\right) \\
& -2 \sum_{l, m} s_{\mu l} w_{l m}^{\mathrm{V}} s_{m v}
\end{aligned}
$$
internuclear distance between atoms $A$ and $B$, respectively. resulting equation is given by

$$
\mathbf{f}^{\mathrm{C}}\left(\mathbf{d}^{\mathrm{C}}\right) \mathbf{c}^{\mathrm{C}}=\mathbf{s}^{\mathrm{C}} \mathbf{c}^{\mathrm{C}} \boldsymbol{\varepsilon}^{\mathrm{C}}
$$

where $Z_{A}, n_{A}^{\mathrm{C}}$, and $R_{A B}$ are the nuclear charge, the number of core electrons in atom $A$, and the

After the valence SCF calculation, the core electron relaxations are performed. For the relaxation, the equation is derived using the above first approximation of Eq. (5) in the FCP method. A similar derivation for the valence-only equation is performed to obtain a core-only equation. The

81 and

$$
w_{\mu v}^{\mathrm{V}}=\sum_{v} \varepsilon_{v} c_{\mu \nu} c_{v v}^{*}
$$

Using the fixed valence orbitals obtained from the valence FCP calculation, the core-only 
energies.

A core energy is defined as

$$
E^{\mathrm{C}}=\frac{1}{2} \sum_{\mu, \nu} d_{\mu \nu}^{\mathrm{C}}\left(h_{\mu \nu}^{\mathrm{C}}+f_{\mu \nu}^{\mathrm{C}}\right)+\sum_{A<B} \frac{\left(Z_{A}-n_{A}^{\mathrm{V}}\right)\left(Z_{B}-n_{B}^{\mathrm{V}}\right)}{R_{A B}}
$$

where $n_{A}^{\mathrm{V}}$ is the number of valence electrons in atom $A$.

\subsection{Implementation}

This subsection gives the implementation of the FCP scheme with relaxations of core electrons (hereinafter called FCP-CR). FCP-CR consists of three steps: an atomic calculation, a valence calculation, and a core calculation as shown in Table 1. In atomic calculations, each atomic AE calculation included in a target molecule is performed to determine the atomic core orbitals and orbital energies. These are then used in the atomic core density matrix in Eq. (4) and the core potential and projection matrices in Eq. (8). In a valence calculation, the valence-only equation in Eq. (7) is solved using the information for the atomic core electrons. Here, valence-valence two-electron integrals, which require smaller basis set dimensions than AE, are evaluated. In addition, the computational scaling for calculations of the core-valence two-electron integrals is theoretically reduced from $O\left(n^{4}\right)$ to $O\left(n^{3}\right)$. Finally, in a core calculation, we perform the SCF calculation based on the core-only equation in Eq. (12) using a fixed valence potential, which is determined using Eq. (12). Note that the core-core and core-valence two-electron integrals with smaller computational costs than the total two-electron integral are evaluated. Throughout 
this Letter, the calculations of the core-valence two-electron integrals for Eq. (12) are skipped because these integrals, which are the same as those used in Eq. (8), are stored on a disk.

\section{Numerical assessments}

\subsection{Computational details}

This section examines the performance of the FCP-CR scheme. The IODKH with LUT (LUTIODKH) Hamiltonian was applied to all the calculations. Numerical assessments were performed to investigate the accuracy and efficiency of the FCP-CR scheme. The Core, valence, and total HF energies, as well as the orbital energies, of coinage metal dimers $\mathrm{M}_{2}(\mathrm{M}=\mathrm{Cu}, \mathrm{Ag}$, and $\mathrm{Au})$ were calculated for accuracy. The bond lengths were 2.55, 2.89, and $2.89 \AA$ for $\mathrm{Cu}_{2}, \mathrm{Ag}_{2}$, and $\mathrm{Au}_{2}$, respectively [30, 31]. The core ionization energies (CIEs) and core level shifts (CLSs) of C 1s and W 4f orbitals were calculated to investigate differences between core electrons in various chemical environments at the $\mathrm{HF}$ and symmetry-adapted cluster configuration interaction (SAC-CI) levels [32,33]. Vinyl acetate was employed for CIEs and CLSs of C 1s orbitals, whose geometry was optimized at the density functional theory (DFT) with the B3LYP functional using cc-pVTZ basis set [34]. In addition, $\mathrm{W}(\mathrm{CO})_{4} \mathrm{~L}[\mathrm{~L}=1$,2-bis(diphenylphosphino)ethane (dppe), 1,2-bis(dimethylphosphino)ethane (dmpe), and 1,2-bis((pentafluorophenyl)phosphino)ethane (F-dppe)] was adopted for CIEs and CLSs of W 4f orbitals. The threshold sets (hartree) for the perturbation selection [35] were $1 \times 10^{-6}$ for SAC and $1 \times 10^{-7}$ for SAC-CI in vinyl acetate and $5 \times 10^{-6}$ for $\mathrm{SAC}$ and $5 \times 10^{-7}$ for $\mathrm{SAC}-\mathrm{CI}$ in $\mathrm{W}(\mathrm{CO})_{4} \mathrm{~L}$ with a non-direct algorithm. Their geometries were 
optimized at DFT with the B3LYP functional using Sapporo-DZP-2012 basis set for main group elements [36] and the SDD relativistic small-core effective core potential for W [37]. Gold clusters were adopted to investigate the efficiency. In these clusters, an experimental lattice constant of $4.062 \AA$ with a face-centered cubic structure was adopted [38]. The total numbers of atoms in the $\mathrm{Au}_{n}$ clusters were $14,32,50,68$, and 86 , which correspond to $1 \times 1 \times 1,1 \times 1 \times 3,1 \times 1 \times 5$, $1 \times 1 \times 7,1 \times 1 \times 9$ unit cells, respectively. In AE, the maximum number of the atoms in a cluster was 50. All the results were compared to those of the AE method.

The basis sets were DKH3-Gen-TK/NOSeC-V-TZP [39-41] for C in vinyl acetate, $\mathrm{Cu}, \mathrm{Ag}$, W, and Au; Sapporo-DZP-2012 for $\mathrm{H}$ and $\mathrm{O}$ in vinyl acetate; and 6-31G [42, 43] for H, C, O, F, and $\mathrm{P}$ in tungsten complexes. In gold clusters the minimal third-order DKH basis set [44] was adopted. Note that a polarization function was added to the basis sets of the $\mathrm{C}$ and $\mathrm{P}$ atoms nearest to the central tungsten atom of these complexes. The valence orbitals were set to $\{2 \mathrm{~s}, 2 \mathrm{p}\}$ for $\mathrm{C}$, $\{3 s, 3 p, 3 d, 4 s\}$ for $\mathrm{Cu},\{4 s, 4 p, 4 d, 5 s\}$ for $\mathrm{Ag},\{5 \mathrm{~s}, 5 \mathrm{p}, 5 \mathrm{~d}\}$ for $\mathrm{W}$, and $\{5 \mathrm{~s}, 5 \mathrm{p}, 5 \mathrm{~d}, 6 \mathrm{~s}\}$ for $\mathrm{Au}$.

HF calculation was performed using our in-house relativistic quantum chemical program. A modified version of the Gaussian09 suite of programs [45] was employed for DFT and SAC-CI calculations.

\subsection{Computational cost of FCP-CR}

This subsection examines the efficiency of FCP-CR. Table 2 shows the CPU times of the HF

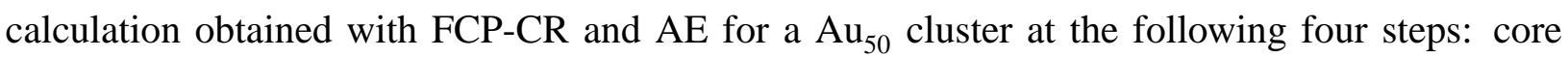


or valence potential (Potential), one-electron integral (OEI), two-electron integral (TEI), and SCF procedure. Valence is the CPU time for the valence electron calculation, which corresponds to FCP, whereas core is the CPU time for the relaxation of core electrons. All is a sum over both the valence and core CPU times. A Xeon E5-2690/2.90 GHz (Octa-core) processor was used on a single core.

The total CPU time of FCP-CR (all) was $34.4 \%$ of that for the AE case: 70816.3 and 203983.8 $s$ for FCP-CR and AE, respectively. The total CPU time was mainly reduced by the TEI and SCF calculations. In the TEI calculation, the number of integrals was decreased by treating only valence or core electrons. The SCF calculation was performed with a smaller dimension basis set in FCP-CR compared with AE. In addition, the CPU time for valence was dominant in FCP-CR compared with that of core.

Figure 1 shows the system-size dependence of the CPU time in $\mathrm{Au}_{n}(n=14,32,50,68$, and 86). The horizontal axis shows the number of gold atoms in the cluster. The vertical axis shows the total CPU time of FCP-CR and AE. FCP-CR reduces the CPU time in comparison with AE. The scalings are $O\left(n^{2.71}\right)$ and $O\left(n^{2.28}\right)$ for AE and FCP-CR, respectively.

To investigate the scaling for FCP-CR, Figure 2 shows the system-size dependence of the CPU time for dominant steps: TEI, the SCF procedure, and construction of a potential (Potential). The CPU times were measured at (a) AE, (b) FCP-CR for valence electrons (equal to FCP), and (c) FCP-CR for core electrons. The scaling of the CPU time in each step is given in Figure 2. In the 
AE, the TEI and SCF scalings are $O\left(n^{2.50}\right)$ and $O\left(n^{4.32}\right)$. Thus, the scaling of the total CPU time becomes $O\left(n^{2.71}\right)$. In FCP-CR, the CPU time for the valence calculation is larger than that for the core calculation. This is because the dimension of the basis set is larger. Furthermore, the TEI steps in the valence and core calculations are dominant. The scalings are $O\left(n^{2.53}\right)$ and $O\left(n^{2.18}\right)$ for valence and core, respectively, and resulting scaling of the total CPU time is $O\left(n^{2.28}\right)$.

\subsection{Accuracy of FCP-CR}

This subsection examines the accuracy of FCP-CR. Table 3 shows the core, valence, and total HF energies (in hartree) obtained with the LUT-IODKH Hamiltonian for $\mathrm{M}_{2}(\mathrm{M}=\mathrm{Cu}, \mathrm{Ag}$, and $\mathrm{Au}$ ), as well as the results of the FCP and molecular AE methods. The deviations from the molecular AEs are shown in parentheses. The energy components of the molecular AE are evaluated using the procedure described in a previous study [28].

The difference in the total energy of FCP is less than 0.09 hartree: $0.001430,0.08759$, and 0.007563 hartree for $\mathrm{Cu}_{2}, \mathrm{Ag}_{2}$, and $\mathrm{Au}_{2}$, respectively. For each molecule, the difference in the core part is smaller than that of the valence part because the core regions are similar to that in the atomic state. On the other hand, the total energies of FCP-CR give small deviations compared with FCP: $0.000188,0.000117$, and 0.001538 hartree for $\mathrm{Cu}_{2}, \mathrm{Ag}_{2}$, and $\mathrm{Au}_{2}$, respectively. The deviations in the valence energy are speculated to be canceled out by those in the core energy because the core electrons are relaxed self-consistently under the frozen valence potential.

Figure 3 shows the orbital energy differences (in hartree) from those of the AE method in 
coinage metal dimers $\left(\mathrm{Cu}_{2}, \mathrm{Ag}_{2}\right.$, and $\left.\mathrm{Au}_{2}\right)$ obtained by FCP and FCP-CR. The vertical axis shows the difference from AE in the orbital energy, $\Delta \varepsilon$. The horizontal axis indicates the orbital species from the core to the valence regions. The dotted line indicates the boundary between the core and valence regions.

The values $\Delta \varepsilon$ in the valence orbitals of FCP-CR are the same as those of FCP because these schemes are common until the valence electrons are solved self-consistently. The mean absolute deviations of both schemes are $0.04,0.04$, and $0.03 \mathrm{eV}$ for $\mathrm{Cu}_{2}, \mathrm{Ag}_{2}$, and $\mathrm{Au}_{2}$, respectively, which is consistent with the results of a previous study [28].

The $\Delta \varepsilon$ values in the core orbitals of FCP-CR are smaller than those of FCP. The mean absolute deviations of FCP-CR are $0.04,0.0$, and $0.02 \mathrm{eV}$ for $\mathrm{Cu}_{2} . \mathrm{Ag}_{2}$, and $\mathrm{Au}_{2}$, respectively, while those of FCP are $0.24,0.22$, and $0.04 \mathrm{eV}$ for $\mathrm{Cu}_{2}, \mathrm{Ag}_{2}$, and $\mathrm{Au}_{2}$, respectively. Thus, the core orbitals can be properly relaxed under a molecular valence potential.

\subsection{Core ionization energy and core level shift}

This subsection discusses the accuracies of the CIEs and CLSs. In HF calculations, the CIEs and CLSs were estimated from the orbital energies based on Koopmans' theorem. In SAC-CI calculations, these values were directly obtained.

Table 4 shows the CIEs (in $\mathrm{eV}$ ) of $\mathrm{C} 1 \mathrm{~s}$ and $\mathrm{W} 4 \mathrm{f}$ in vinyl acetate and $\mathrm{W}(\mathrm{CO})_{4} \mathrm{~L}$ complexes, respectively. Vinyl acetate has four types of $\mathrm{C} 1 \mathrm{~s}$ with different chemical environments: COO, $\mathrm{CHO}, \mathrm{CH}_{3}$, and $\mathrm{CH}_{2}$. In addition, $\mathrm{W}(\mathrm{CO})_{4} \mathrm{~L}$ has three types of $\mathrm{W} 4 \mathrm{f}$ with different ligands: dppe, 
dmpe, and F-dppe. The CLSs (in eV), which are shown in parentheses, were estimated using the differences from the reference values. As references, $\mathrm{CH}_{3}$ and dppe were adopted for vinyl acetate and $\mathrm{W}(\mathrm{CO})_{4} \mathrm{~L}$, respectively. The experimental values for vinyl acetate and $\mathrm{W}(\mathrm{CO})_{4} \mathrm{~L}$ were taken from Refs. 46 and 47, respectively.

In FCP, the CIEs at the HF level were always the same value in different chemical environments: $308.298 \mathrm{eV}$ and $47.540 \mathrm{eV}$ for vinyl acetate and $\mathrm{W}(\mathrm{CO})_{4} \mathrm{~L}$, respectively. On the other hand, in FCP-CR, the CIEs depended on the chemical environment. The CIEs and CLSs were close to those of AE, within a deviation of $0.5 \mathrm{eV}$. Furthermore, the electron correlation effect, which is the difference between $\mathrm{HF}$ and SAC-CI, contributed to reproducing the values of the experimental CIEs. The deviations from the experimental CIEs were less than 5.7 and $6.0 \mathrm{eV}$ for vinyl acetate and $\mathrm{W}(\mathrm{CO})_{4} \mathrm{~L}$, respectively. Note that the deviations from the experimental values are mainly caused by the incompleteness of the basis sets, which is shown in Appendix B.

In vinyl acetate, the tendency is explained by the characteristics of the atoms or bonds surrounding a target atom. For $\mathrm{COO}$, the carbon has bonds with two electronegative oxygen atoms, which leads to a decrease of the electron density at $\mathrm{C}$. For $\mathrm{CHO}$, the shift is smaller than that of $\mathrm{COO}$ because $\mathrm{CHO}$ has one $\mathrm{C}-\mathrm{O}$ bond. For $\mathrm{CH}_{2}$, the carbon has one double bond with a hybridized $\mathrm{sp}^{2}$ orbital. The hybridization expands the valence orbitals compared with the $\mathrm{sp}^{3}$ hybridization in $\mathrm{CH}_{3}$, which also leads to a decrease of the electron density at $\mathrm{C}$.

In $\mathrm{W}(\mathrm{CO})_{4} \mathrm{~L}$, the tendency is explained by the characteristics of the ligands. For dppe, the shift 
is smaller than that of dmpe because of the screening capability of a core hole in the ligands. The $\pi$ electrons of the phenylphosphine group are likely polarized in comparison with the $\sigma$ electrons of the methylphosphine group, which leads to easy screening of core holes [47]. For F-dppe, the pentafluorophenylphosphine group includes highly electronegative $\mathrm{F}$, and thus the electron density at $\mathrm{W}$ decreases.

\section{Concluding remarks}

In the present study, we have proposed the FCP-CR scheme for relaxation of core electrons based on FCP at the IODKH level. The core-only HFR equation is derived using the same procedure as that for a valence-only one. The molecular core orbitals and their energies are computed under the frozen valence molecular potential, which is constructed on the fly. Numerical assessments were performed with coinage metal dimers and small gold clusters to examine accuracy and efficiency. The CIEs and CLSs of C $1 \mathrm{~s}$ in vinyl acetate and $\mathrm{W} 4 \mathrm{f}$ in three tungsten complexes were calculated with FCP-CR at the HF and SAC-CI levels. The results suggest that FCP-CR sufficiently reproduces the corresponding AE results with high efficiency. The proposed scheme has a high potential for becoming an alternative and efficient AE method, without loss of accuracy.

\section{Acknowledgments}

We performed some of the calculations in the present study at the Research Center for Computational Sciences (RCCS), Okazaki Research Facilities, National Institutes of Natural Sciences 
(NINS). This study was supported in part by the Ministry of Education Culture, Sports, Science and Technology (MEXT), Japan; the MEXT program "Elements Strategy Initiative to Form Core Research Center" (since 2012), MEXT, Japan; and the Core Research for Evolutional Science and on Relativistic Electronic Theory" of the Japan Science and Technology Agency (JST). 


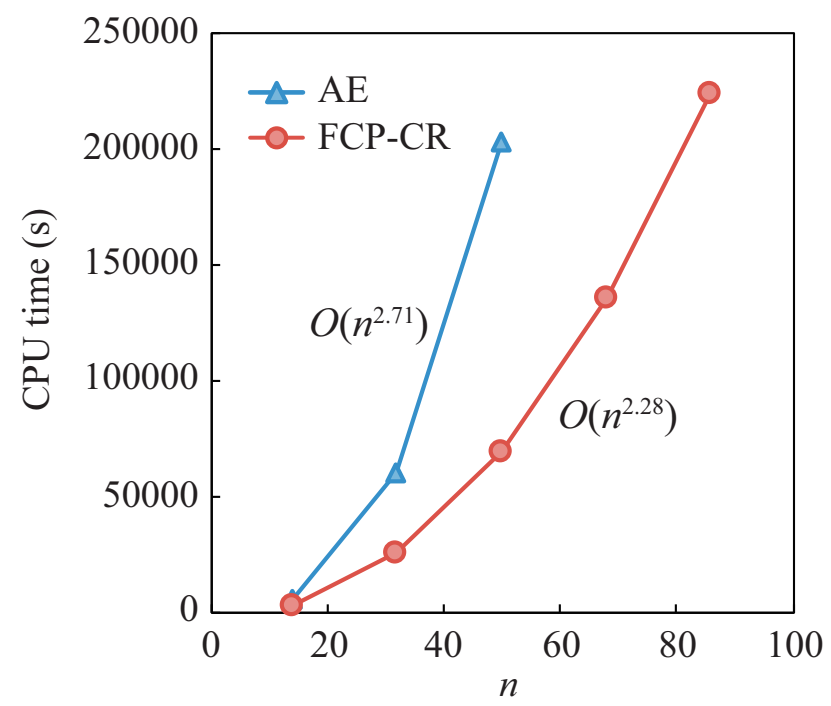

Figure 1: System-size dependence of CPU time (s) in $\mathrm{Au}_{n}(n=14,32,50,68$, and 86) obtained with the AE and FCP-CR methods. A Xeon E5-2690/2.90 GHz (octa-core) processor was used on a single core.
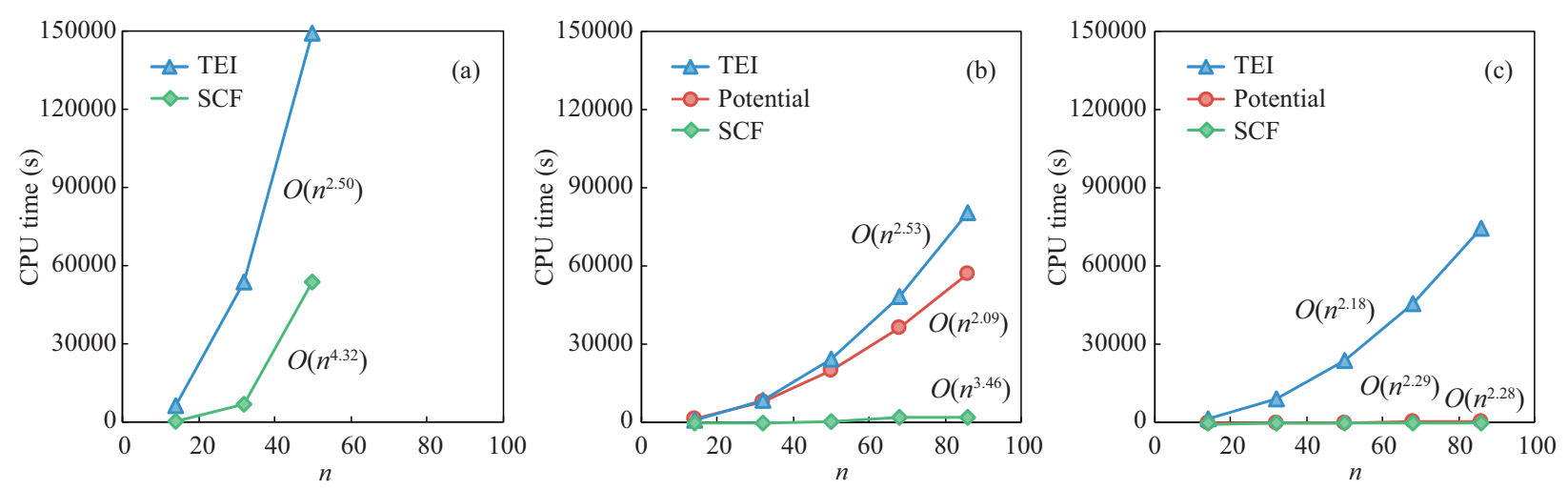

Figure 2: System-size dependence of CPU time (s) for dominant steps with the AE and FCP-CR methods in $\mathrm{Au}_{n}(n=$ 14, 32, 50, 68, and 86): two-electron integral (TEI), SCF procedure, and construction of potential (Potential). (a) AE; (b) valence part in FCP-CR; and (c) core part in FCP-CR. A Xeon E5-2690/2.90 GHz (octa-core) processor was used on a single core. 


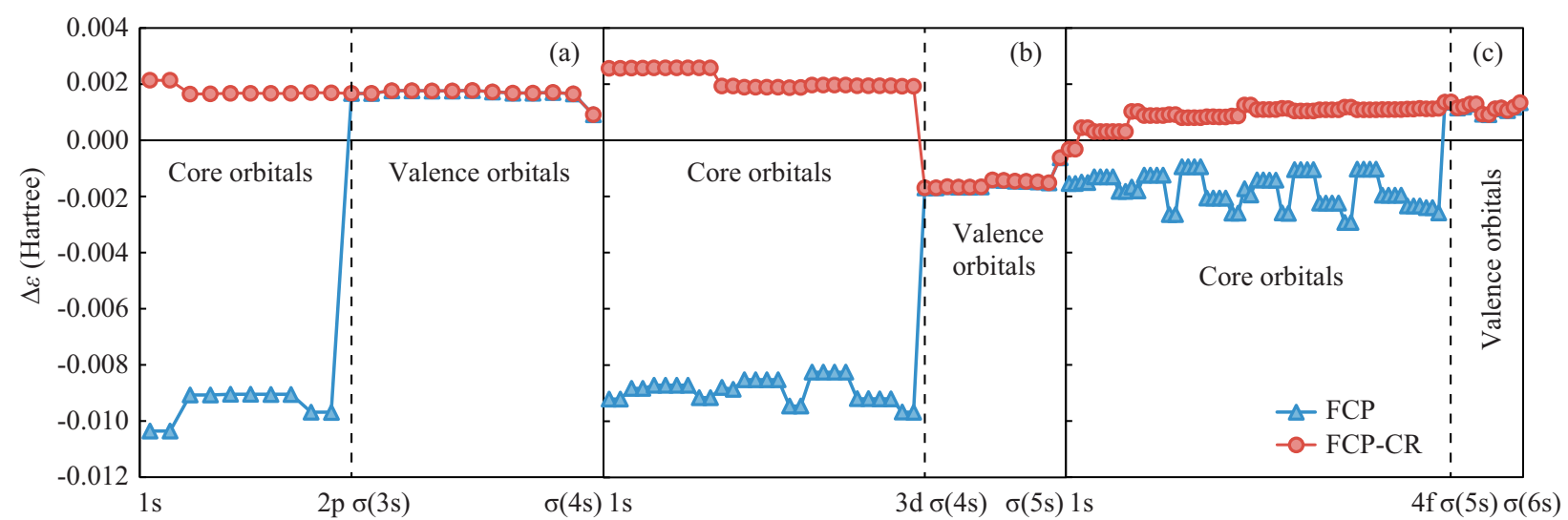

Figure 3: Differences of between the FCP and FCP-CR orbital energies (in hartree) and those of the AE method in coinage metal dimers at the Hartree-Fock level: (a) $\mathrm{Cu}_{2}$, (b) $\mathrm{Ag}_{2}$, and (c) $\mathrm{Au}_{2}$. The orbitals are sorted in ascending order of energy. 
(I) Atomic calculations (I.1) One- and two-electron integrals for each atom

(I.2) All-electron HF calculation for each atom

(I.3) Save core orbital, density matrix, and orbital energy

(II) Valence calculation (II.1) Core-valence two-electron integrals of third term of r.h.s. in Eq. (8)

(II.2) Construct core potential

(II.3) Construct projection operator matrices of fourth term of r.h.s. in Eq. (8)

(II.4) One- and two-electron integrals for valence

(II.5) Add projection operator matrix and core potential

(II.6) Solve valence-only equation of Eq. (7)

(III) Core calculation

(III.1) Construct fixed valence potential of second term of r.h.s. in Eq. (12)

(III.2) Construct projection operator matrices in Eq. (12)

(III.3) One- and two-electron integrals for core

(III.4) Add projection operator matrix and fixed valence potential

(III.5) Solve core-only equation of Eq. (11) 
Table 2: CPU time for four steps in the Hartree-Fock calculation obtained with FCP-CR and AE for a $\mathrm{Au}_{50} \mathrm{cluster}$ construction of core or valence potential (Potential), one-electron integral (OEI), two-electron integral (TEI), and SCF procedure (SCF). A Xeon E5-2690/2.90 GHz processor (octa-core) was used on a single core. The percentages of individual steps are given in parentheses.

\section{FCP-CR}

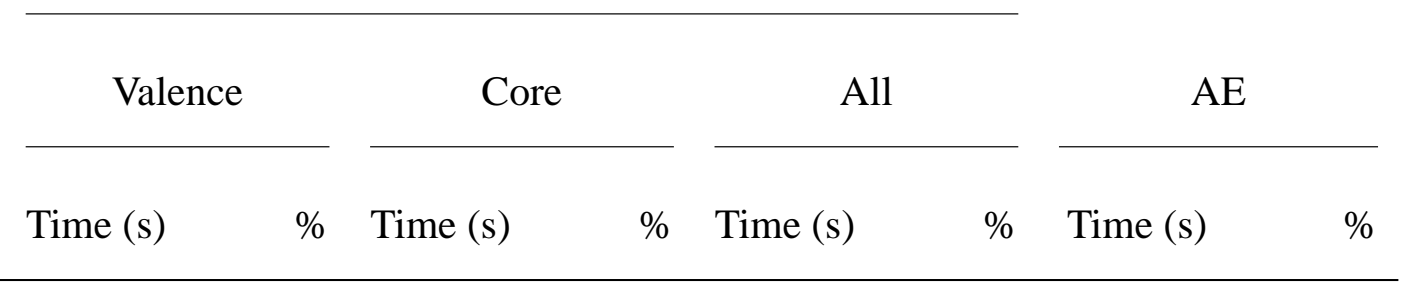

\begin{tabular}{rrrrrrrrrr} 
Potential & 20120.4 & $(44.5)$ & 175.9 & $(0.7)$ & 20296.3 & $(28.9)$ & 0.0 & $(0.0)$ \\
OEI & 328.4 & $(0.7)$ & 719.0 & $(2.9)$ & 1047.4 & $(1.5)$ & 985.1 & $(0.5)$ \\
TEI & 24363.1 & $(53.8)$ & 23991.0 & $(96.2)$ & 48354.1 & $(68.9)$ & 149132.0 & $(73.1)$ \\
SCF & 440.0 & $(1.0)$ & 48.5 & $(0.2)$ & 488.5 & $(0.7)$ & 53866.7 & $(26.4)$ \\
Total & 45251.9 & $(100.0)$ & 24934.4 & $(100.0)$ & 70186.3 & $(100.0)$ & 203983.8 & $(100.0)$ \\
\hline
\end{tabular}


Table 3: Comparison of core, valence, and total $\mathrm{HF}$ energies (in hartree) of $\mathrm{M}_{2}(\mathrm{M}=\mathrm{Cu}, \mathrm{Ag}$, and $\mathrm{Au}$ ) obtained by the FCP, FCP-CR, and molecular AE methods. The deviations from the molecular AEs are given in parentheses.

\begin{tabular}{|c|c|c|c|c|c|c|}
\hline & & FCP & & FCP-CR & & Molecular AE \\
\hline \multirow[t]{3}{*}{$\mathrm{Cu}_{2}$} & Core & -2913.534822 & $(-0.000063)$ & -2913.533204 & $(0.001555)$ & -2913.534759 \\
\hline & Valence & -392.856146 & $(-0.001367)$ & -392.856146 & $(-0.001367)$ & -392.854779 \\
\hline & Total & -3306.390968 & $(-0.001430)$ & -3306.389350 & $(0.000188)$ & -3306.389538 \\
\hline \multirow[t]{3}{*}{$\mathrm{Ag}_{2}$} & Core & -10332.790532 & $(-0.000150)$ & -10332.704656 & $(0.085726)$ & -10332.790382 \\
\hline & Valence & -293.119642 & $(-0.085609)$ & -293.119642 & $(-0.085609)$ & -293.034033 \\
\hline & Total & -10625.910174 & $(-0.085759)$ & -10625.824298 & $(0.000117)$ & -10625.824415 \\
\hline \multirow[t]{3}{*}{$\mathrm{Au}_{2}$} & Core & -37750.131480 & $(0.001660)$ & -37750.137505 & $(-0.004365)$ & -37750.133140 \\
\hline & Valence & -272.254111 & (0.005903) & -272.254111 & $(0.005903)$ & -272.260014 \\
\hline & Total & -38022.385591 & $(0.007563)$ & -38022.391616 & $(0.001538)$ & -38022.393154 \\
\hline
\end{tabular}


Table 4: Core ionization energies (in eV) obtained by FCP, FCP-CR, and AE of C 1s and W 4f for vinyl acetate and three tungsten complexes, respectively. Experimental values are taken from Ref. 46 for vinyl acetate and Ref. 47 for the tungsten complexes. Core level shifts from the reference molecules $\left(\mathrm{CH}_{3}\right.$ or dppe) are given in parentheses.

\begin{tabular}{|c|c|c|c|c|c|c|c|}
\hline \multirow[b]{2}{*}{ Molecule } & \multirow[b]{2}{*}{ type } & \multicolumn{3}{|c|}{$\mathrm{HF}$} & \multicolumn{2}{|c|}{ SAC-CI } & \multirow[b]{2}{*}{ Exptl. } \\
\hline & & $\mathrm{FCP}$ & FCP-CR & $\mathrm{AE}$ & FCP-CR & $\mathrm{AE}$ & \\
\hline \multirow[t]{8}{*}{ vinyl acetate } & $\mathrm{COO}$ & 308.298 & 310.249 & 310.103 & 298.617 & 298.475 & 294.94 \\
\hline & & (0.000) & (3.951) & (3.938) & (3.870) & $(3.860)$ & (3.510) \\
\hline & $\mathrm{CHO}$ & 308.298 & 307.700 & 307.553 & 295.726 & 295.585 & 292.32 \\
\hline & & $(0.000)$ & (1.402) & (1.388) & $(0.979)$ & $(0.970)$ & $(0.890)$ \\
\hline & $\mathrm{CH}_{3}$ & 308.298 & 306.298 & 306.165 & 294.747 & 294.615 & 291.43 \\
\hline & & $(0.000)$ & $(0.000)$ & $(0.000)$ & $(0.000)$ & $(0.000)$ & $(0.000)$ \\
\hline & $\mathrm{CH}_{2}$ & 308.298 & 305.634 & 305.509 & 293.181 & 293.061 & 290.54 \\
\hline & & (0.000) & $(-0.664)$ & $(-0.656)$ & $(-1.566)$ & $(-1.554)$ & $(-0.890)$ \\
\hline \multirow[t]{6}{*}{$\mathrm{W}(\mathrm{CO})_{4} \mathrm{~L}$} & dppe & 47.540 & 47.589 & 48.033 & 36.274 & 36.558 & 31.118 \\
\hline & & (0.000) & $(0.000)$ & $(0.000)$ & $(0.000)$ & $(0.000)$ & $(0.000)$ \\
\hline & dmpe & 47.540 & 47.588 & 48.030 & 36.963 & 37.236 & 31.296 \\
\hline & & (0.000) & $(-0.001)$ & $(-0.003)$ & (0.689) & (0.678) & $(0.178)$ \\
\hline & F-dppe & 47.540 & 48.316 & 48.773 & 37.403 & 37.742 & 31.931 \\
\hline & & $(0.000)$ & $(0.727)$ & $(0.740)$ & (1.129) & (1.184) & $(0.813)$ \\
\hline
\end{tabular}




\section{Appendix A. Accuracy of an iterative procedure between valence and core calculations}

This appendix examines the effect of an iterative self-consistent procedure between the valence and core calculations. FCP-CR calculates core orbitals under a valence potential only once, after valence orbitals are calculated under an atomic core potential. However, the valence orbitals could be more accurate if the valence orbitals were recalculated under the core potential obtained by FCP-CR. Here, a procedure is iteratively performed between the valence and core calculations based on Eqs. (7), (8), (11), and (12). Table A.1 shows the iteration number dependence in total energy (hartree) of $\mathrm{Au}_{2}$. Iteration $=0$ and 1 indicate FCP and FCP-CR, respectively. $\Delta E$ is the deviation in the total energy from the previous value. $\Delta E^{\mathrm{AE}}$ is the deviation from the reference value obtained by molecular AE; the reference value is -38022.393154 hartree. The total energy was steeply converged with a small iteration number. The largest deviation in $\Delta E^{\mathrm{AE}}$ is 0.001456 hartree at the fifth iteration. This deviation is due to the approximation of the basis set in Eq. (5). Furthermore, the largest deviation in $\Delta E$ is -0.006025 hartree at the first iteration, which is the difference between FCP and FCP-CR. This result indicates that FCP-CR, which requires only one calculations of core orbitals under a valence potential, is enough to describe the core electrons in a chemical environment. 
Table A.1: Iteration number dependence in total energy (in hartree) of $\mathrm{Au}_{2}$ obtained by the valence-core iterative procedure.

\begin{tabular}{ccrc}
\hline Iteration & Energy & $\Delta E$ & $\Delta E^{\mathrm{AE}}$ \\
\hline $0(\mathrm{FCP})$ & -38022.385591 & - & 0.007563 \\
$1(\mathrm{FCP}-\mathrm{CR})$ & -38022.391616 & -0.006025 & 0.001537 \\
2 & -38022.391693 & -0.000077 & 0.001461 \\
3 & -38022.391697 & -0.000004 & 0.001457 \\
4 & -38022.391698 & -0.000001 & 0.001456 \\
5 & -38022.391698 & 0.000000 & 0.001456 \\
\hline
\end{tabular}




\section{Appendix B. Dependence of core ionization potential energies}

We examined core ionization potential energies by the standard SAC-CI calculations without a symmetry $\left(C_{1}\right)$ as well as using different basis sets: cc-pCVXZ $(X=\mathrm{D}, \mathrm{T}$, and $\mathrm{Q})$ and special basis sets designed for a core ionization potential in the paper written by Kuramoto and Ehara [J. Chem. Phys. 122 (2005) 014304]. Table B.2 shows the core ionization energies (eV) of C 1s for vinyl acetate. $\Delta$ shows the difference from the experimental values. In the $C_{1}$ symmetry, $\Delta$ is almost the same as the $C_{\mathrm{S}}$ symmetry in Section 3. In the cc-pCVXZ types of basis sets, $\Delta$ gets systematically smaller as the cardinal number $X$ increases. For cc-pCVQZ basis set, the maximum deviation is $1.34 \mathrm{eV}$ compared with $4.93 \mathrm{eV}$ for cc-pCVDZ and 1.55 for cc-pCVTZ. Moreover, the special basis set gives the best results among these basis sets. The maximum deviation is $1.04 \mathrm{eV}$ although the size of the basis sets is smaller than cc-pCVQZ, because the special basis set includes the differential basis function for the primitive s-type of function. These indicate that the proper basis set selection requires for reproducing the experimental values. 


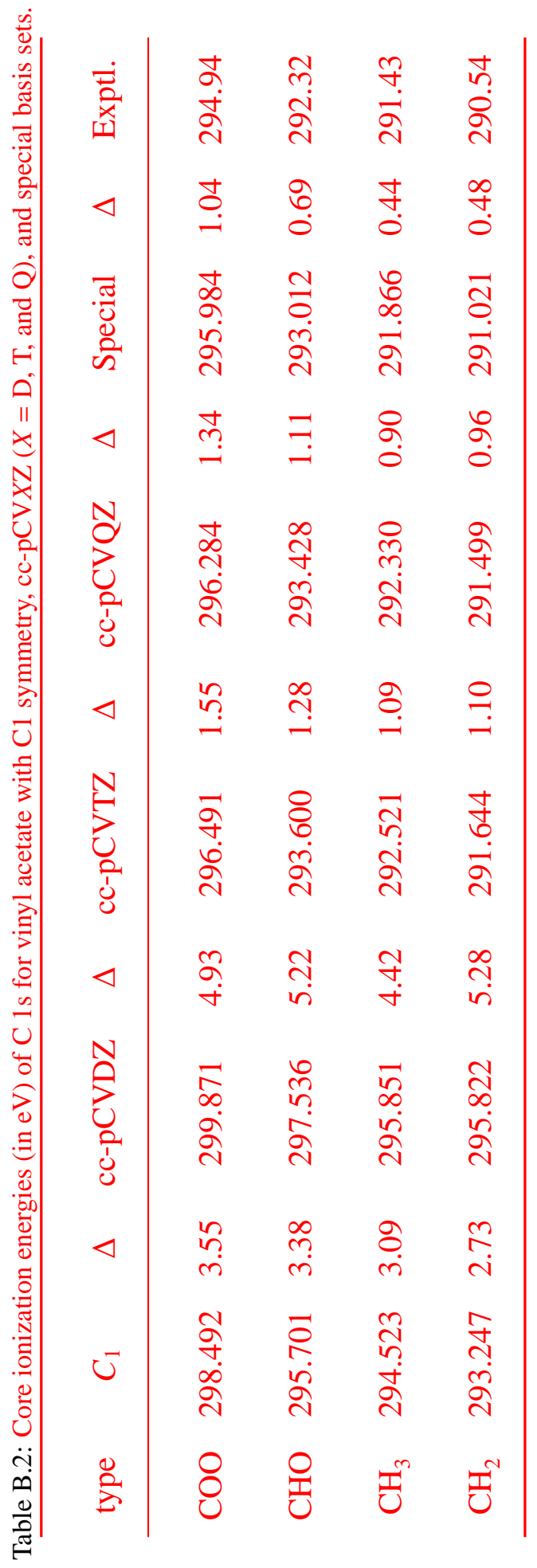




\section{References}

[1] R. E. Moss, Advanced Molecular Quantum Mechanics, Chapman and Hall, London, 1973.

[2] T. Nakajima, M. Yanai, K. Hirao, J. Comput. Chem. 23 (2002) 847.

[3] M. S. Kelly, T. Shiozaki, J. Chem. Phys. 138 (2013) 204113.

[4] T. Shiozaki, J. Chem. Theory Comput. 9 (2013) 4300.

[5] K. G. Dyall, J. Chem. Phys. 106 (1997) 9618.

[6] W. Liu, D. Peng, J. Chem. Phys. 125 (2006) 044102.

[7] M. Barysz, A. J. Sadlej, J. Chem. Phys. 116 (2002) 2696.

[8] U. Wahlgren, B. Schimmelpfennig, S. Jusuf, H. Strömsnes, O. Gropen, L. Maron, Chem. Phys. Lett. 287 (1998) 525.

[9] J. Thar, B. Kirchner, J. Chem. Phys. 130 (2009) 124103.

[10] J. Seino, H. Nakai, J. Chem. Phys. 136 (2012) 244102.

[11] J. Seino, H. Nakai, J. Chem. Phys. 137 (2012) 144101.

[12] J. Seino, H. Nakai, J. Chem. Phys. 139 (2013) 034109.

[13] J. Seino, H. Nakai, Int. J. Quantum Chem. 115 (2015) 253.

[14] J. Seino, H. Nakai, J. Comput. Chem. Jpn. 13 (2014) 1.

[15] D. Peng, M. Reiher, J. Chem. Phys. 136 (2012) 244108.

[16] D. Peng, N. Middendorf, F. Weigend, M. Reiher, J. Chem. Phys. 138 (2013) 184105.

[17] J. C. Phillips, L. Kleinman, Phys. Rev. 116 (1959) 287.

[18] J. D. Weeks, S. A. Rice, J. Chem. Phys. 49 (1968) 2741.

[19] D. R. Hamann, M. Schlüter, C. Chiang, Phys. Rev. Lett. 43 (1979) 1494.

[20] P. J. Hay, W. R. Wadt, J. Chem. Phys. 82 (1985) 270.

[21] D. Vanderbilt, Phys. Rev. B 41 (1990) 7892. 
[22] K. Lassonen, A. Pasquarello, R. Car, C. Lee, D. Vanderbilt, Phys. Rev. B 47 (1993) 10142.

[23] D. Figgen, G. Rauhut, M. Dolg, H. Stoll, Chem. Phys. 311 (2005) 227.

[24] Y. Sakai, E. Miyoshi, S. Klobukowski, M. Huzinaga, J. Comput. Chem. 8 (1987) 226.

[25] F. Rakowitz, C. M. Marian, L. Seijo, J. Chem. Phys. 111 (1999) 10346.

[26] T. Tsuchiya, T. N. Nakajima, K. Hirao, L. Seijo, Chem. Phys. Lett. 361 (2002) 334.

[27] T. Zeng, M. Klobukowski, J. Chem. Phys. 130 (2009) 204107.

[28] J. Seino, M. Tarumi, H. Nakai, Chem. Phys. Lett. 592 (2014) 341.

[29] S. Huzinaga, A. A. Cantu, J. Chem. Phys. 55 (1971) 5543.

[30] T. Nautiyal, S. Youn, K. Kim, Phys. Rev. B 68 (2003) 033407.

[31] G. Nazin, X. Qiu, W. Ho, Phys. Rev. Lett. 90 (2003) 216110.

[32] H. Nakatsuji, K. Hirao, J. Chem. Phys. 68 (1978) 2053.

[33] H. Nakatsuji, Chem. Phys. Lett. 67 (1979) 334.

[34] T. H. Dunning, Jr., J. Chem. Phys. 90 (1989) 1007.

[35] H. Nakatsuji, Chem. Phys. 75 (1983) 425.

[36] T. Noro, M. Sekiya, T. Koga, Theor. Chem. Acc. 131 (2012) 1124.

[37] D. Figgen, K. A. Peterson, M. Dolg, H. Stoll, J. Chem. Phys. 130 (2009) 164108.

[38] P. Haas, F. Tran, P. Blaha, Phys. Rev. B 79 (2009) 085104.

[39] H. Tatewaki, T. Koga, Chem. Phys. Lett. 328 (2000) 473.

[40] M. Sekiya, T. Noro, Y. Osanai, T. Koga, Theor. Chem. Acc. 106 (2001) 297.

[41] Y. Osanai, T. Noro, E. Miyoshi, M. Sekiya, T. Koga, J. Chem. Phys. 120 (2004) 6408.

[42] W. J. Hehre, R. Ditchfield, J. A. Pople, J. Chem. Phys. 56 (1972) 2257.

[43] J. D. Dill, J. A. Pople, J. Chem. Phys. 62 (1975) 2921.

[44] T. Tsuchiya, M. Abe, T. Nakajima, K. Hirao, J. Chem. Phys. 115 (2001) 4463. 
[45] M. J. Frisch, G. W. Trucks, H. B. Schlegel, G. E. Scuseria, M. A. Robb, J. R. Cheeseman, G. Scalmani, V. Barone, B. Mennucci, G. A. Petersson, H. Nakatsuji, M. Caricato, X. Li, H. P. Hratchian, A. F. Izmaylov, J. Bloino, G. Zheng, J. L. Sonnenberg, M. Hada, M. Ehara, K. Toyota, R. Fukuda, J. Hasegawa, M. Ishida, T. Nakajima, Y. Honda, O. Kitao, H. Nakai, T. Vreven, J. A. Montgomery, Jr., J. E. Peralta, F. Ogliaro, M. Bearpark, J. J. Heyd, E. Brothers, K. N. Kudin, V. N. Staroverov, R. Kobayashi, J. Normand, K. Raghavachari, A. Rendell, J. C. Burant, S. S. Iyengar, J. Tomasi, M. Cossi, N. Rega, J. M. Millam, M. Klene, J. E. Knox, J. B. Cross, V. Bakken, C. Adamo, J. Jaramillo, R. Gomperts, R. E. Stratmann, O. Yazyev, A. J. Austin, R. Cammi, C. Pomelli, J. W. Ochterski, R. L. Martin, K. Morokuma, V. G. Zakrzewski, G. A. Voth, P. Salvador, J. J. Dannenberg, S. Dapprich, A. D. Daniels, Ö. Farkas, J. B. Foresman, J. V. Ortiz, J. Cioslowski, D. J. Fox, Gaussian 09 Revision D.01, Gaussian Inc. Wallingford CT, 2009.

[46] A. A. Bakke, A. W. Chen, W. L. Jolly, J. Electron Spectrosc. Relat. Phenom. 20 (1980) 333.

[47] C. Crotti, E. Farnetti, T. Celestino, M. Stener, S. Fontana, Organometallics 23 (2004) 5219. 
Graphical Abstract
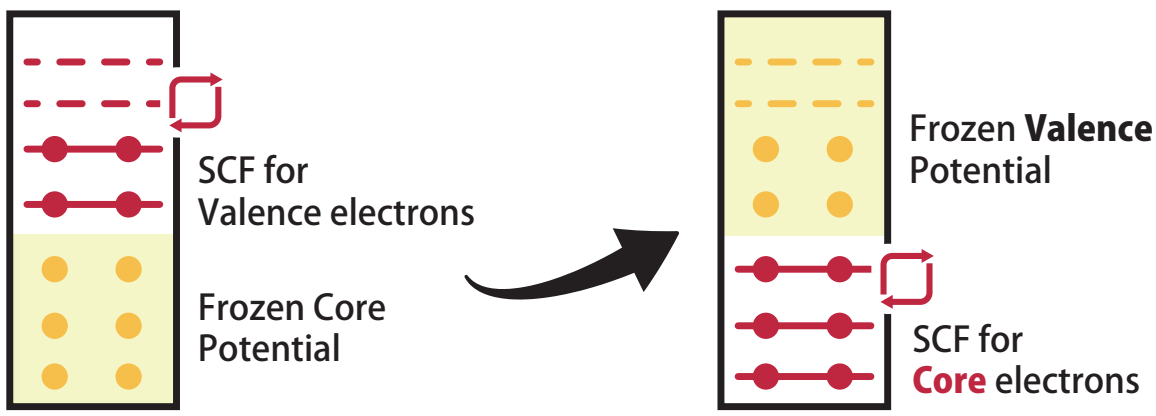

FCP

FCP-CR 